Session 2532

\title{
Introductory Electric Circuits on the Internet via Mallard ${ }^{\circledR}$ Software
}

\author{
Hans H. Kuehl \\ University of Southern California
}

\begin{abstract}
The introductory sophomore linear circuits course at the University of Southern California was taught for the first time via the Internet in the spring of 1999, using Mallard $®$ software to deliver the homework assignments. Mallard ${ }^{\circledR}$ was chosen because it has many attractive features that make it well suited to support the teaching of an introductory circuits course.
\end{abstract}

The student response to the use of Mallard ${ }^{\circledR}$ was extremely positive. Student interest in the homework was increased markedly. Especially liked by students was the capability to submit homework solutions at any hour of the day or night via the Internet, to get immediate feedback regarding the correctness of their answers, and to be able to submit a revised answer if the previous answer was incorrect

In the development of the homework assignments, we have structured the more involved Mallard $®$ problems to contain a series of steps requiring intermediate answers. This allows the student to more easily pinpoint where the error lies if the final solution is incorrect.

This paper describes our experiences in this initial endeavor, and focuses primarily on:

1. The advantages of using Mallard ${ }^{\circledR}$ in teaching an introductory circuits course.

2. The response of the students to use of Mallard $®$ in the teaching of electric circuits.

3. Our approach in the development of problems with a structure that helps to guide the student along the path toward the correct solution.

\section{Introduction}

EE 202 is a basic sophomore linear circuits course at the University of Southern California (USC) with a large enrollment consisting of both electrical and biomedical engineering students, which is well suited for Web-based enhancements and asynchronous learning networks teaching/learning techniques. Because of this, we have developed a new version of this course that incorporates an innovative Web-based learning technology that we find strengthens student interest in learning the course material. The principal thrust of this initial effort was the development of Web-based homework problems for this course using Mallard ${ }^{\circledR}$ software. ${ }^{1}$ We have been successful in fulfilling our fundamental goal, which was to have it on-line in the spring of 1999, making it the first engineering course at USC that has interactive Web-based homework using Mallard $\circledast$ courseware. Based on student response and performance, the Mallard $\AA$-based enhancement of our basic circuits course has been very successful. The Mallard ${ }^{\circledR}$ platform and format appear to have great appeal to our students, increasing their interest and performance significantly on the homework assignments. In this paper, the 
advantages of the use of Mallard $\AA$, the development and coding of the homework problems, and the student response are described.

\section{Advantages of Using Mallard ${ }^{\circledR}$}

We chose Mallard $\AA$, which was developed at the University of Illinois, ${ }^{1}$ as the platform for our Web-enhanced version of EE 202 at USC. The University of Illinois has been a pioneer in the innovative use of the Web in education. A course somewhat similar to our EE 202 is taught using Mallard $\AA$ at the University of Illinois by Prof. Tim Trick. Another innovative approach that uses the Web to teach electric circuits, which is capable of many of the features of Mallard $\AA$, is called CircuitTutor ${ }^{\circledR}{ }^{2}$ and was implemented at the University of Illinois by Prof. Burks Oakley, II. ${ }^{3}$ It should be noted that Prof. Robert M Anderson, Jr. at Iowa State University already began to successfully use Mallard ${ }^{\circledR}$ in 1997 to administer and deliver the homework in his introductory electric circuits course, and has now been sharing and extending the instructional material for several semesters. ${ }^{4,5}$

Of primary importance in EE 202 is that the students learn the basic principles, which include Ohm's law, Kirchhoff's voltage and current laws, and the associated techniques necessary to analyze and design analog circuits. It is also very important that students practice these techniques by doing many homework problems. One of the reasons for choosing Mallard® over other software systems was that Mallard ${ }^{\circledR}$ has the mathematical and computational functionality necessary to evaluate and grade the homework problems in an introductory engineering course such as EE 202. Mallard ${ }^{\circledR}$ gives the course developer both power and flexibility in constructing homework questions by making available several pre-programmed question formats or modules, called input types, to generate questions. These input types include fill-in-the-blank, arithmetic, multiple choice, and equation evaluation. In EE 202, we utilized primarily the arithmetic and equation evaluation types. In the arithmetic input type, the student is required to submit a numerical answer, which is then evaluated and graded by Mallard $\circledast$. In the equation evaluation input type, the student submits a mathematical expression, which is then evaluated and graded by Mallard ${ }^{\circledR}$. This is an especially valuable feature in that it allows the evaluation and grading of algebraic expressions in symbolic form. In addition, Mallard ${ }^{\circledR}$ allows random values to be programmed into problems so that individual students typically get different numerical values in their problem statements. Furthermore, students do not need any special software on their machines; only a standard Internet browser (e.g. Netscape or Microsoft Internet Explorer) is required of students. Mallard ${ }^{\circledR}$ graded problem scores are automatically recorded; other student grades (e.g., examination scores, paper-and-pencil homework scores, laboratory scores) can be uploaded. Each student can thus know all his/her own scores and how his/her scores compare with the entire class.

All of the homework in the course is done over the Web; students access the homework problems and submit their solutions via Mallard ${ }^{\circledR}$ at any hour of the day or night. Our current "computer generation" students are very enthusiastic about this new way to do homework. A primary reason for this increased enthusiasm is that when they submit their solutions via their computers, Mallard $\AA$ immediately grades the problems and returns the result without delay on the monitor screen. Thus, they have immediate feedback when their work is freshest in their 
minds and do not have to wait a week or two until their graded papers are returned with no opportunity to submit a corrected solution. I allow them to resubmit answers up to 10 times before the due date without penalty so that, if submitted solutions are incorrect, they can rethink their solution methods and try again. This encourages students to try to solve each problem perfectly, which has resulted in most of the resulting homework grades being relatively high. This process is very beneficial to the learning process and it is gratifying to note that these high grades suggest that the increased student interest in the homework due to Mallard ${ }^{\circledR}$ translates into an increased comprehension of the principles on which the homework is based.

\section{Problem Coding}

As indicated above, by means of Mallard $\circledast$, students use computers to both access the homework problems and submit their answers. Mallard $\AA$ immediately grades the problems and returns the grades on the monitor screen. This is accomplished in the Mallard® system by means of a special coding of the problems using an extended version of HTML. This extended version of HTML contains all the standard HTML tags but has, in addition, other tags that enable Mallard® to produce features such as the various question input types (fill-in-the blank, arithmetic, true/false, multiple choice, equation evaluation, etc.), random values for variables in the problem statement, as well as immediate grading. The main initial task in our development of the Mallard ${ }^{\circledR}$-based version of EE 202 (and probably in any engineering or science course) was to create and code the homework problems into the Mallard ${ }^{\circledR}$ extended HTML format. To save time, we chose to base our homework problems on many of the existing excellent problems in our textbook, ${ }^{6}$ although, as described subsequently, considerable modification was required for the more complex and involved problems. Because we used the textbook problems, it was necessary to obtain permission from the textbook authors as well as the publisher to adapt the problems for use in the Web-based course.

In the development and coding of our Mallard ${ }^{\circledR}$ problems, we have utilized the following guidelines to facilitate the student learning process:

(a) If Mallard ${ }^{\circledR}$ marks the student's submitted answer to a problem incorrect, sufficient feedback should be available to the student to help determine the source of the error. In the case of a problem for which only a relatively simple calculation is required to obtain the answer, however, feedback is generally not called for because it is usually not difficult for the student to determine where the error in his/her calculation lies and submit a revised answer.

(b) It is generally detrimental to the learning process and discouraging to the student to have full credit for a more complex problem depend entirely on one final answer because, if that answer is incorrect, it is difficult for the student to determine where the mistake has occurred. To mitigate this problem, the more involved Mallard ${ }^{\circledR}$ problems should be structured to contain a series of steps requiring intermediate answers. This allows the student to more easily pinpoint where the error lies if the final solution is incorrect.

(c) Small coding errors can cause Mallard $\AA$ to tell the student that his/her correct answer is incorrect. Therefore, every effort should be made to ensure that the coding of each problem is error-free.

A team of two graduate students and one undergraduate student provided help in the construction and coding of problems. The simplest problems could be created and coded in a few minutes 
whereas many of the more involved problems often required several hours before an acceptable version was constructed and perfected. Before embarking on the project, the student assistants knew some HTML but had to spend a few hours to become familiar with the additional elements of the Mallard ${ }^{\circledR}$ extended version before they could begin coding problems.

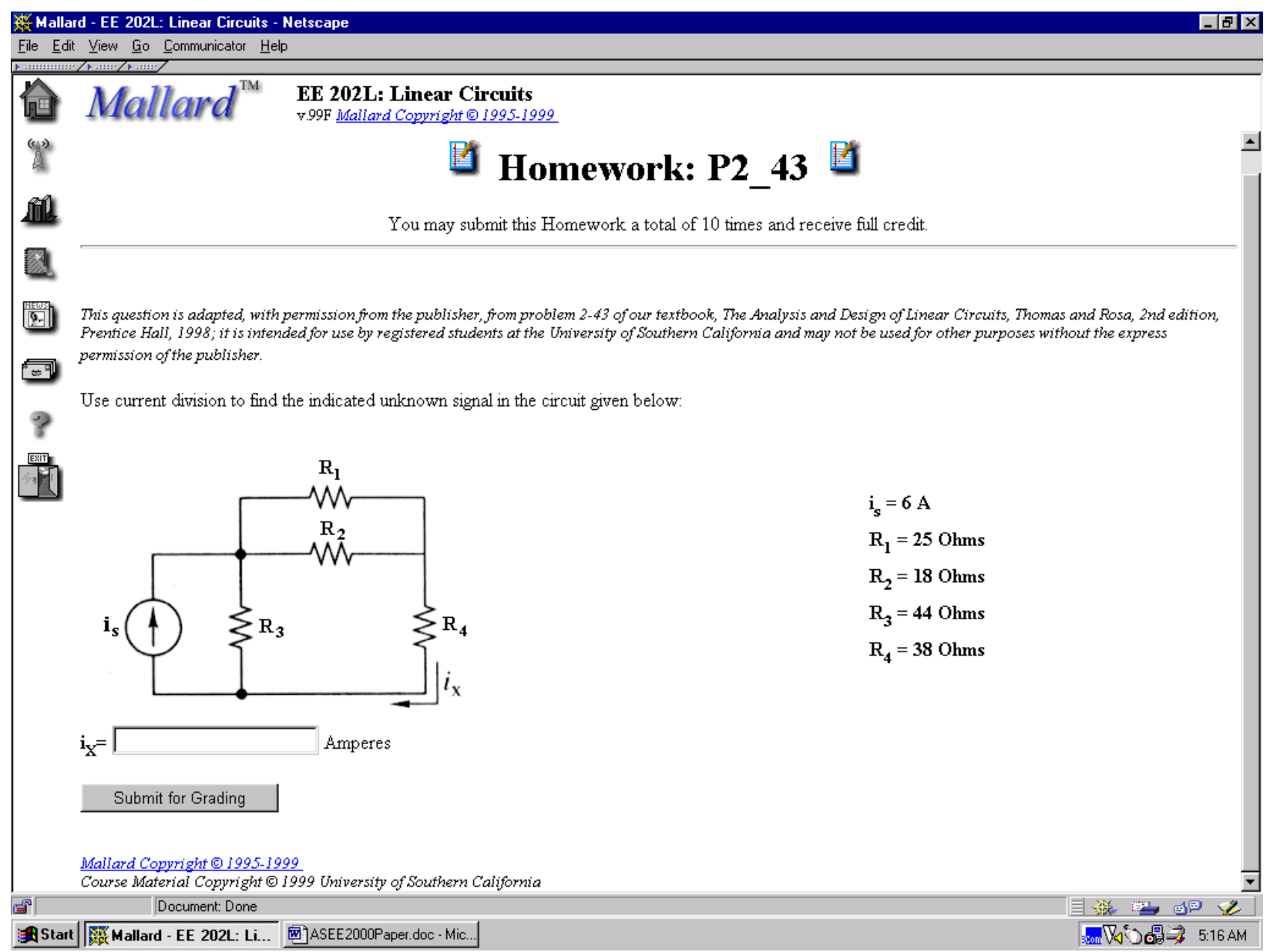

Figure 1 Student view of a relatively simple problem (P2_43).

Shown in Figure 1 is an example of what a student sees when he/she accesses a homework problem. The problem shown is relatively straightforward in that it requires the student to carry out only a simple calculation to obtain the answer. Accordingly, the short problem statement shown is an essentially unmodified version of that in the textbook; ${ }^{6}$ we have inserted no additional intermediate steps to help the student locate any error in his/her calculation because, if the student's submitted answer is graded incorrect by Mallard ${ }^{\circledR}$, it should not be difficult for the student to rework the problem, find the error and submit a corrected answer. The only modification made to the textbook version of this problem is the assignment of random values to the circuit parameters, i.e., in the textbook the values of $i_{s}, R_{1}, R_{2}$, etc. on the circuit schematic are shown as numerical values, whereas in the Mallard ${ }^{\circledR}$ schematic we have replaced these numerical values by the symbols $i_{s}, R_{1}, R_{2}$, etc. The coding of the problem is such that the numerical values of these parameters are assigned random values by Mallard $\AA$, which are shown 
beside the schematic. Thus, individual students typically get different parameter values in their problem statements so, although they are able to help each other with the solution approach, they are not able to share numerical answers. Also shown at the beginning of the problem is the "permission statement" that the publisher of the textbook, ${ }^{6}$ Prentice Hall, and we have agreed would be included.

Shown below is the source code for this relatively simple problem:

Question P2_43, Version 1:

$\langle\mathrm{P}\rangle\langle\mathrm{I}\rangle<$ FONT SIZE $=-1>$ This question is adapted, with permission from the publisher, from problem 2-43 of our textbook, The Analysis and Design of Linear Circuits, Thomas and Rosa, 2nd edition, Prentice Hall, 1998; it is intended for use by registered students at the University of Southern California and may not be used for other purposes without the express permission of the publisher. $</$ FONT $></ \mathrm{I}>$

$</ \mathrm{P}\rangle$

$\langle\mathrm{P}\rangle$

Use current division to find the indicated unknown signal in the circuit given below:

$</ \mathrm{P}\rangle$

$\langle$ TABLE WIDTH=90\%>

$\langle\mathrm{TR}>$

$\langle\mathrm{TD}\rangle$

$<$ img width=335 height=223 src=figp2-43c1m.gif $>$

$</ \mathrm{TD}>$

$<\mathrm{TD}>$

$\langle$ TABLE $>$

$\langle\mathrm{TR}\rangle\langle\mathrm{TD}\rangle$

$\left\langle\mathrm{B}>\mathrm{i}\langle\mathrm{SUB}\rangle_{\mathrm{s}}\langle/ \mathrm{SUB}\rangle=\langle\right.$ random name $=$ I1 range $=-10-10$ type=int nonzero $>\mathrm{A}<\mathrm{B}\rangle$

$\langle/ \mathrm{TD}\rangle\langle/ \mathrm{TR}\rangle$

$\langle\mathrm{TR}\rangle\langle\mathrm{TD}\rangle$

$\langle\mathrm{B}\rangle \mathrm{R}\langle\mathrm{SUB}\rangle 1</ \mathrm{SUB}\rangle=\langle$ random name $=\mathrm{R} 1$ range $=10-50$ type=int $>$ Ohms $\langle/ \mathrm{B}\rangle$

$\langle/ \mathrm{TD}\rangle\langle/ \mathrm{TR}\rangle$

$\langle\mathrm{TR}\rangle\langle\mathrm{TD}\rangle$

$\langle\mathrm{B}\rangle \mathrm{R}\langle\mathrm{SUB}\rangle 2\langle/ \mathrm{SUB}\rangle=\langle$ random name $=\mathrm{R} 2$ range $=10-50$ type $=$ int $>\mathrm{Ohms}\langle/ \mathrm{B}\rangle$

$\langle/ \mathrm{TD}\rangle\langle/ \mathrm{TR}\rangle$

$\langle\mathrm{TR}\rangle\langle\mathrm{TD}\rangle$

$\langle\mathrm{B}\rangle \mathrm{R}\langle\mathrm{SUB}\rangle 3\langle/ \mathrm{SUB}\rangle=\langle$ random name $=\mathrm{R} 3$ range $=10-50$ type $=$ int $>\mathrm{Ohms}</ \mathrm{B}\rangle$

$\langle/ \mathrm{TD}\rangle\langle/ \mathrm{TR}\rangle$

$\langle\mathrm{TR}\rangle\langle\mathrm{TD}\rangle$

$\langle\mathrm{B}>\mathrm{R}\langle\mathrm{SUB}>4</ \mathrm{SUB}\rangle=\langle$ random name $=\mathrm{R} 4$ range $=10-50$ type $=$ int $>$ Ohms $</ \mathrm{B}>$ $\langle/ \mathrm{TD}\rangle\langle/ \mathrm{TR}\rangle$

$</$ TABLE $>$

$\langle/ \mathrm{TD}\rangle$

$</ \mathrm{TR}\rangle$

$</$ TABLE $>$

$<$ eval name=Ra noshow $>$

$\langle$ var name=R1 $>*\langle$ var name=R2 $>/(\langle$ var name=R1 $>+\langle$ var name=R2 $\rangle)+\langle$ var name=R4 $\rangle$ 
$</$ eval $>$

$\langle\mathrm{B}>\mathrm{i}<\mathrm{SUB}\rangle \mathrm{X}</ \mathrm{SUB}\rangle=</ \mathrm{B}>$

$<$ input type $=$ arith tolerance $=1 \%>$

$<$ input $>$

$<$ answer $>$

$\langle$ var name $=$ I1 $\rangle *\langle$ var name $=\mathrm{R} 3\rangle /(\langle$ var name $=\mathrm{Ra}\rangle+\langle$ var name $=\mathrm{R} 3\rangle)$

$</$ answer $>$

Amperes

Browse Included Images: figp2-43c1m.gif

This source code includes examples of several of the types of available Mallard $\AA$ extended HTML tags that give the programmer considerable flexibility in achieving desired formats for both the problem statement and the answer submission. The <random>tags that assign random values to $i_{s}, R_{1}, R_{2}$, etc. are exhibited; they also contain the desired range of these values, as well as their specification (in this case) as integers. Between the <eval> and </eval> tags is an expression that is evaluated numerically for later use in obtaining the final answer. In this case, the evaluated number will not be displayed in the problem statement because of the "noshow" at the end of the <eval> tag. The <input> tag determines the question type, which in this case specifies that the type of answer to be submitted by the student is to be arithmetic, i.e., numerical, and that the tolerance is $1 \%$, i.e., the numerical value submitted by the student will be marked correct if it is within $1 \%$ of the exact correct answer. Contained between the <answer> and </answer> tags is the formula that calculates the correct answer to the problem, which Mallard $®$ uses to determine if the student's submitted answer is within the specified tolerance. After submission of an answer, Mallard $\circledast$ informs the student if it is correct or not.

Figure 2 shows an example of a more complicated problem (with answers inserted) structured to contain a series of steps requiring intermediate answers. This problem was adapted from a textbook problem ${ }^{6}$ that simply asked for a single answer, the equation for the output voltage $v_{0}(t)$. Because this final answer is the result of a rather lengthy calculation, we inserted intermediate steps, each of which require intermediate answers to be submitted, in order to break the problem into several parts. In this way, the student can more easily pinpoint any error he/she has made if the submitted answer for $v_{0}(t)$ is marked incorrect by Mallard $\circledast$. The rather lengthy source code for this problem is shown in the Appendix. The arithmetic answer format, used in the previous example, is used only in part (a) of this problem. Parts (b), (c), and (d) contain the very useful "equation evaluation" format, whereby the student's submitted answer is in the form of a mathematical expression that can contain one independent variable (in this case, the time variable $\mathrm{t}$ ), as well as simple mathematical functions such as the unit step, trigonometric, logarithmic, and exponential functions. Thus, this format allows questions that require symbolic answers, which is one of the most valuable and attractive features of Mallard $\circledast$.

It should be noted that the view of the problem shown in Fig. 2 is not what a student sees when accessing this problem. Near the bottom of Fig. 2 are two buttons labeled "Check Answer" and "Cheat," which are accessible only to the "Course Director" or "Course Developer." The "Check Answer" button allows the "Course Director" or "Course Developer" to determine the correctness of any answers entered in the answer boxes of the problem, whereas activation of the 
"Cheat" button enters the correct answers in the answer boxes. In the student version of the problem a single button labeled "Submit for Grading" as shown in Fig. 1 replaces the "Check Answer" and "Cheat" buttons.

Figure 3 shows the response of Mallard ${ }^{\circledR}$ when the answers shown in Fig. 2 are submitted for grading by using the "Check Answer" button. A student would receive the same response to these answers but would also receive a homework grade summary giving grade information regarding this and any previous submissions of this problem.

\section{Student Response}

The students in the class generally were very enthusiastic about this new way to do homework. In all the years that I have taught at USC, I have never received as much student interest and response (e-mail, office visits, phone calls) regarding the homework as I did during this first "Mallard ${ }^{\circledR}$ semester." I received e-mail at all hours of the day and night from students doing the Mallard ${ }^{\circledR}$ homework with questions and comments regarding the problems. Although some comments were from students who thought their incorrect answers were correct even though Mallard $®$ said they were wrong, I was not concerned, as long as Mallard $®$ intensified their interest in doing the homework! A primary reason for this increased enthusiasm is evidently the immediate grading by Mallard $\AA$ of students' submitted answers. Thus, they have immediate feedback and don't have to wait a week or two until their graded papers are returned. Because I allow them to resubmit answers up to 10 times before the due date without penalty, it gives them the opportunity, if a submitted solution is incorrect, to rethink their solution methods while they are fresh in their minds and try again. It is gratifying to note that the high grades on the homework suggest that the increased student interest in the homework due to Mallard ${ }^{\circledR}$ translates into an increased comprehension of the principles on which the homework is based.

Several students expressed to me their excitement to be part of this new experiment in asynchronous learning. A couple of the students told me that they even got their parents (in other states) $\operatorname{logged}$ in to the Mallard ${ }^{\circledR}$ Website to show them this innovative way to do homework. We also have the Mallard ${ }^{\circledR}$ Website linked to the EE 202 Newsgroup and homepage.

Near the end of the semester, the students completed an evaluation of the use of Mallard® in the course. Some of the results are:

96\% of the students liked the immediate grading of problems (They don't have to wait a week or more for graded homework to be handed back).

92\% liked being able to resubmit a solution up to 10 times without penalty before the due date (They learn from their mistakes by reworking problems while they are still fresh in their minds).

85\% think that Mallard $\circledR$ should be continued in EE 202. 


\section{Question P7_46, Version 1}

This question is adapted, with permission from the publisher, from problem 7 - 46 of our textbook, The Analysis and Design of Linear Circuits, Thomas and Rosa, 2nd edition, Prentice Hall, 1998; it is intended for use by registered students at the University of Southern California and may not be used for other purposes without the express permission of the publisher.

How to express your answer

The circuit shown is in the zero state when the step function input is applied.

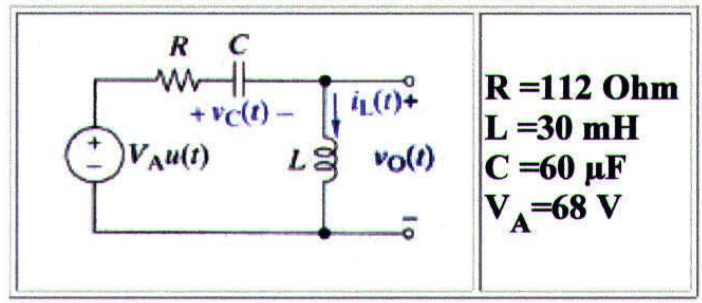

(a) Find the two roots, $s_{1}$ and $s_{2}$, of the characteristic equation. Let $s_{1}$ be the larger root.

\begin{tabular}{|c|c|c|}
\hline$s_{1}=$ & -155.267 & $\mathrm{~s}^{-1}$ \\
\hline$s_{2}=$ & -3578.066 & $\mathrm{~s}^{-1}$ \\
\hline
\end{tabular}

(b) Find $v_{C}(t)$ for $t \geq 0$.

$\boldsymbol{v}_{\boldsymbol{C}}(\mathbf{t})=-71.08 * \exp (-155.267 * t)+3.085 * \exp (-3578.066 * t)+68$

(c) Find $\boldsymbol{i}_{\boldsymbol{L}}(\mathbf{t})$ for $\mathbf{t} \geq \mathbf{0}$.

$\boldsymbol{i}_{\boldsymbol{L}}(\mathbf{t})=0.6622 *(\exp (-155.267 * t)-\exp (-3578.066 * t))$ A

(d) Find $v_{o}(t)$ for $\mathbf{t} \geq \mathbf{0}$.

$\boldsymbol{v}_{\boldsymbol{O}}(\mathbf{t})=19.87 *(-155.3 * \exp (-155.267 * t)--3578.1 * \exp (-3578.066 * t)) \mathbf{V}$

Check Answer Cheat

\section{Question Course Material Quiz Multimedia Upload Browsers Access Managers}

Mallard Copyright C 1995-1999

Course Material Copyright Copyright University of Southern California

Figure 2 A problem with several parts to help guide the student to the final answer (P7_46). Answers have been inserted in the answer boxes. 


\section{Question P7_46, Version 1}

This question is adapted, with permission from the publisher, from problem 7 - 46 of our textbook, The Analysis and Design of Linear Circuits, Thomas and Rosa, 2nd edition, Prentice Hall, 1998; it is intended for use by registered students at the University of Southern California and may not be used for other purposes without the express permission of the publisher.

How to express your answer

The circuit shown is in the zero state when the step function input is applied.

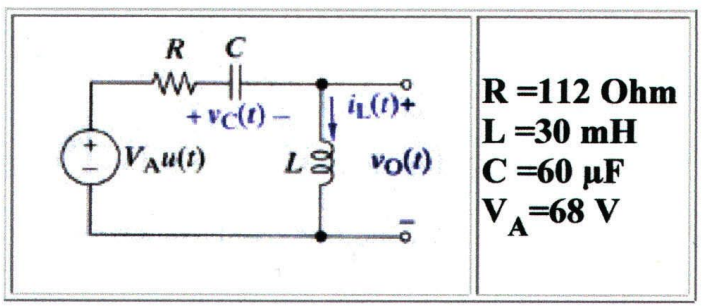

(a) Find the two roots, $s_{1}$ and $s_{2}$, of the characteristic equation. Let $s_{1}$ be the larger root.

$$
\begin{array}{|l|}
\hline s_{1}=-155.267 \\
s_{2}=-3578.066 \text { s } \mathrm{s}^{-1} \\
\hline
\end{array}
$$

(b) Find $v_{C}(t)$ for $\mathbf{t} \geq \mathbf{0}$.

$$
v_{C}(t)=-71.08 * \exp (-155.267 * t)+3.085 * \exp \left(-3578.066^{*} t\right)+68 \text { } \mathrm{V}
$$

(c) Find $i_{L}(\mathbf{t})$ for $\mathbf{t} \geq \mathbf{0}$.

$$
i_{L}(t)=0.6622 *(\exp (-155.267 * t)-\exp (-3578.066 * t)) \text { 田 }
$$

(d) Find $\boldsymbol{v}_{\boldsymbol{O}}(\mathbf{t})$ for $\mathbf{t} \geq \mathbf{0}$.

$$
v_{O}(t)=19.87 *\left(-155.3 * \exp (-155.267 * t)--3578.1^{*} \exp \left(-3578.066^{*} t\right)\right) \text { } V
$$

Item 1: Your answer is within $\pm 1 \%$ of the solution - correct.

Item 2: Your answer is within $\pm 1 \%$ of the solution - correct.

Item 3: Your equation is within $1 \%$ of the solution!

Item 4: Your equation is within $1 \%$ of the solution!

Item 5: Your equation is within $1 \%$ of the solution!

You received a raw score of $100 \%$ on this question.

\section{Question Course Material Quiz Multimedia Upload Browsers Access Managers}

Mallard Copyright (C) 1995-1999

Course Material Copyright Copyright University of Southern California

Figure 3 Showing Mallard ${ }^{\circledR}$ response to the submission of the answers shown in Fig. 2. 


\section{Summary and Conclusions}

The introductory sophomore linear circuits course at the University of Southern California was taught for the first time via the Internet in the spring of 1999 , using Mallard ${ }^{\circledR}$ software to deliver and grade the homework assignments. Mallard ${ }^{\circledR}$ was chosen because it has many attractive features that make it well suited to support the teaching of an introductory circuits course. These features include:

a) Mathematical and computational functionality that is adequate to evaluate and grade the homework problems.

b) Pre-programmed question types from which the course developer can choose.

c) No special software required of the student; only a standard Internet browser is needed.

d) Students' submitted answers can be numerical or algebraic in symbolic form.

e) Capability to program random values into problems so that individual students typically get different numerical values in their problem statements.

f) Immediate evaluation and grading of submitted problem solutions and return of the result without delay at any hour of the day or night.

g) Mallard $®$ graded problem scores are automatically recorded; other student grades (e.g., examination scores, paper-and-pencil homework scores, laboratory scores) can be uploaded.

The main initial task in the development of a Mallard®-based version of an introductory engineering or science course is generally to create and code the homework problems into the Mallard ${ }^{\circledR}$ extended HTML format. For problems not requiring extensive calculation by the student, this is usually a straightforward task. The creation and coding of more involved problems, which require extended calculations to arrive at a final answer, is more complex and time consuming. In such problems, it is beneficial to the student learning process for the problem developer to structure the problem to contain several steps that require intermediate answers. This allows the student to more easily pinpoint where the error lies if the final solution is incorrect and submit a corrected answer.

The student response to the use of Mallard $\AA$ in our introductory circuits course was extremely positive. Student interest in the homework was increased markedly. Especially liked by students was the capability to submit homework solutions at any hour of the day or night via the Internet, to get immediate feedback regarding the correctness of their answers, and to be able to submit a revised answer if the previous answer was incorrect

Appendix. Mallard source code for problem P7_46

Question P7_46, Version 1:

$\langle\mathrm{P}\rangle\langle\operatorname{div}$ align=justify $>$

$<\mathrm{I}\rangle\langle$ FONT SIZE $=-1>$ This question is adapted, with permission from the publisher, from problem 7 - 46 of our textbook, The Analysis and Design of Linear Circuits, Thomas

and Rosa, 2nd edition, Prentice Hall, 1998; it is intended for use by registered students at the University of Southern California and may not be used for other purposes without the express permission of the publisher. $\langle/$ FONT $\rangle\langle/$ I $\rangle$ $</ \operatorname{div}></ \mathrm{P}>$ 
$<$ link src=test window=hint link_text="How to express your answer"

title="Express your answer" $>$

$<\mathrm{P}\rangle$

The circuit shown is in the zero state when the step function input is applied.

$</ \mathrm{P}>$

<!-- image -->

$\langle$ TABLE BORDER $=1>$

$\langle\mathrm{TR}\rangle$

$\langle$ TD $\rangle\langle$ img width=202 height=124 src=figP7_46.gif $\rangle\langle/ \mathrm{TD}\rangle$

$\langle\mathrm{TD}\rangle\langle\mathrm{P}\rangle$

$<\mathrm{B}>$

$\mathrm{R}=\langle$ random name $=\mathrm{R}$ range $=112-120$ type $=\mathrm{int}\rangle \mathrm{Ohm}\langle\mathrm{BR}\rangle$

$\mathrm{L}=\langle$ random name $=\mathrm{L}$ range $=25-75$ type $=$ int $>\mathrm{mH}\langle\mathrm{BR}>$

$\mathrm{C}=\langle$ random name $=\mathrm{C}$ range $=25-75$ type $=$ int $>\mu \mathrm{F}\langle\mathrm{BR}>$

$\mathrm{V}\langle\mathrm{SUB}\rangle \mathrm{A}\langle/ \mathrm{SUB}\rangle=\langle$ random name $=\mathrm{VA}$ range $=10-100$ type $=$ int $\rangle \mathrm{V}\langle\mathrm{BR}\rangle$ $</ \mathrm{B}>$

$\langle/ \mathrm{TD}>$

$</ \mathrm{TR}>$

$</$ TABLE $>$

$<\mathrm{P}\rangle$

(a) Find the two roots, $\langle$ B $\rangle\langle\text { I }\rangle_{\text {S }}\langle$ SUB $>1</$ SUB $\rangle\langle$ I $\rangle\langle/$ B $>$ and $\left\langle\right.$ B $>\langle\text { I }\rangle_{\text {S }}<$ SUB $>2</$ SUB $\rangle\langle/$ I $\rangle</$ B $>$,

of the characteristic equation. Let $\left.\langle\mathrm{B}\rangle\langle\mathrm{I}\rangle_{\mathrm{S}}\langle\mathrm{SUB}\rangle 1</ \mathrm{SUB}\right\rangle\langle/ \mathrm{I}\rangle\langle/ \mathrm{B}\rangle$ be the larger root. $</ \mathrm{P}>$

$<$ !-- calculations -->

$<$ eval name $=\mathrm{X}$ noshow $>$

$<$ var name $=\mathrm{R}>*<$ var name $=\mathrm{C}>* 1 \mathrm{e}-6$

$</$ eval $>$

<eval name $=$ Y noshow $>$ $\operatorname{sqrt}\left(<\right.$ var name $=\mathrm{R}>\wedge 2 *<$ var name $=\mathrm{C}>\wedge 2^{*} * 1 \mathrm{e}-12-4 *\langle$ var name $=\mathrm{L}\rangle *\langle$ var name $\left.=\mathrm{C}\rangle * 1 \mathrm{e}-9\right)$ $</$ eval $>$

<eval name $=\mathrm{Z}$ noshow $>$

$2 *<$ var name $=\mathrm{L}>*<$ var name $=\mathrm{C}>* 1 \mathrm{e}-9$

$</$ eval $>$

$<$ eval name $=\mathrm{S} 1$ noshow $>$

$(-\langle$ var name $=X\rangle+\langle$ var name $=\mathrm{Y}\rangle) /\langle$ var name $=\mathrm{Z}\rangle$ 
$</$ eval $>$

<eval name $=\mathrm{S} 2$ noshow $>$

$(-\langle$ var name $=\mathrm{X}\rangle-\langle$ var name $=\mathrm{Y}\rangle) /\langle$ var name $=\mathrm{Z}\rangle$

$</$ eval $>$

$<!--$ answer boxes -->

$\langle\mathrm{P}\rangle$

$\langle$ TABLE BORDER $=1>$

$\langle\mathrm{TR}\rangle$

$\langle\mathrm{TD}\rangle$

$\left\langle\right.$ B $>\langle\text { I }\rangle_{\text {S }}\langle$ SUB $>1</$ SUB $\rangle\langle/$ I $\rangle=$

$<$ input type=arith tolerance $=1 \%>$

$<$ input $>$

$<$ answer>

$<$ var name $=$ S1 $>$

$</$ answer $>$ s $<$ SUP $>-1</$ SUP $></$ B $>$

$</ \mathrm{TD}>$

$\langle/ \mathrm{TR}\rangle$

$\langle\mathrm{TR}\rangle$

$\langle\mathrm{TD}\rangle$

$\left\langle\right.$ B $>\langle\text { I }\rangle_{\text {s }}<$ SUB $>2</$ SUB $></$ I $>=$

$<$ input type=arith tolerance $=1 \%>$

$<$ input $>$

$<$ answer>

$<$ var name $=$ S2 $>$

$</$ answer $>$ s $<$ SUP $>-1</$ SUP $></$ B $>$

$\langle/ \mathrm{TD}>$

$</ \mathrm{TR}>$

$</$ TABLE $>$

$</ \mathrm{P}>$

$<\mathrm{P}>$

(b) Find $\langle$ B $\rangle\langle$ I $>$ v $\langle$ SUB $>$ C $</$ SUB $\rangle\langle$ II $\rangle$ (t) $\langle$ /B $>$ for $\langle$ B $>$ t $\langle$ SUB $\rangle$

$<$ img width $=17$ height $=20 \mathrm{src}=$ Gteq. gif $\rangle\langle/ \mathrm{SUB}\rangle 0</ \mathrm{B}\rangle .\langle\mathrm{BR}\rangle$

$<$ !-- calculations -->

<eval name $=\mathrm{K} 1$ noshow $>$

$-\langle$ var name $=\mathrm{VA}\rangle /(1-\langle$ var name=S1 $>/\langle$ var name=S2 $\rangle)$

$</$ eval $>$

<eval name $=\mathrm{K} 2$ noshow $>$

$\langle$ var name $=\mathrm{VA}\rangle *(\langle$ var name $=\mathrm{S} 1\rangle \mid\langle$ var name $=\mathrm{S} 2\rangle) /(1-\langle$ var name $=\mathrm{S} 1\rangle \mid\langle$ var name $=\mathrm{S} 2\rangle)$

$</$ eval> 
$<!--$ answer box -->

$\langle\mathrm{B}\rangle\langle\mathrm{I}\rangle \mathrm{v}<\mathrm{SUB}\rangle \mathrm{C}</ \mathrm{SUB}\rangle\langle/ \mathrm{I}\rangle(\mathrm{t})=$

$<$ input type=equation_eval size $=55>$

$\langle$ param name $=$ indep_var value $=\mathrm{t}\rangle$

$\langle$ param name $=$ check_points value $=0.1,-1 /\langle$ var name $=\mathrm{S} 1\rangle,-1 /\langle$ var name $=\mathrm{S} 2\rangle\rangle$

$\langle$ param tolerance $=1 \%>$

$<$ input>

$<$ answer>

$<$ var name $=\mathrm{K} 1>* \exp (<\operatorname{var}$ name $=\mathrm{S} 1\rangle * \mathrm{t})+<\operatorname{var}$ name $=\mathrm{K} 2\rangle * \exp (<\operatorname{var}$ name $=\mathrm{S} 2\rangle * \mathrm{t})+<$ var name $=\mathrm{VA}>$

$</$ answer $>\mathrm{V}</ \mathrm{B}>$

$</ \mathrm{P}>$

$<\mathrm{P}\rangle$

(C) Find $\langle$ B $>\langle$ I $>$ i $<$ SUB $>$ L $</$ SUB $></$ I $>$ (t) $</$ B $>$ for $\langle$ B $>$ t $<$ SUB $>$

$<$ img width $=17$ height $=20 \mathrm{src}=$ Gteq. gif $\rangle\langle/ \mathrm{SUB}\rangle 0</ \mathrm{B}\rangle .\langle\mathrm{BR}\rangle$

$<!--$ calculations -->

<eval name $=\mathrm{K} 3 \_1$ noshow $>$

$\langle$ var name $=\mathrm{C}\rangle *\langle$ var name $=\mathrm{S} 1\rangle *\langle$ var name $=\mathrm{S} 2\rangle *\langle$ var name $=\mathrm{VA}\rangle * 1 \mathrm{e}-6$

$</$ eval $>$

$<$ eval name $=\mathrm{K} 3$ noshow $>$

$<$ var name $=\mathrm{K} 3 \_1>/(<$ var name $=\mathrm{S} 1\rangle-\langle$ var name $\left.=\mathrm{S} 2\rangle\right)$

$</$ eval $>$

$<!--$ answer box -->

$<\mathrm{B}>\langle\mathrm{I}>\mathrm{i}<\mathrm{SUB}>\mathrm{L}</$ SUB $></ \mathrm{I}>(\mathrm{t})=$

$<$ input type=equation_eval size $=55>$

$<$ param name $=$ indep_var value $=\mathrm{t}>$

$\langle$ param name $=$ check_points value $=0,-1 /\langle$ var name $=\mathrm{S} 1\rangle,-1 /\langle$ var name $=\mathrm{S} 2\rangle\rangle$

$\langle$ param tolerance $=1 \%>$

$<$ input $>$

$<$ answer >

$\langle$ var name $=\mathrm{K} 3>*(\exp (<\operatorname{var}$ name $=\mathrm{S} 1>* \mathrm{t})-\exp (\langle\operatorname{var}$ name $=\mathrm{S} 2\rangle * \mathrm{t}))$

$</$ answer $>\mathrm{A}</ \mathrm{B}>$

$</ \mathrm{P}>$

$\langle\mathrm{P}\rangle$

(d) Find $\langle$ B $\rangle\langle$ I $>$ v $<$ SUB $>$ O $</$ SUB $\rangle<$ I $>$ (t) $<$ /B $>$ for $\langle$ B $>$ t $\langle$ SUB $>$

$<$ img width $=17$ height $=20 \mathrm{src}=$ Gteq. gif $\rangle\langle/ \mathrm{SUB}\rangle 0\langle/ \mathrm{B}\rangle .\langle\mathrm{BR}\rangle$ 


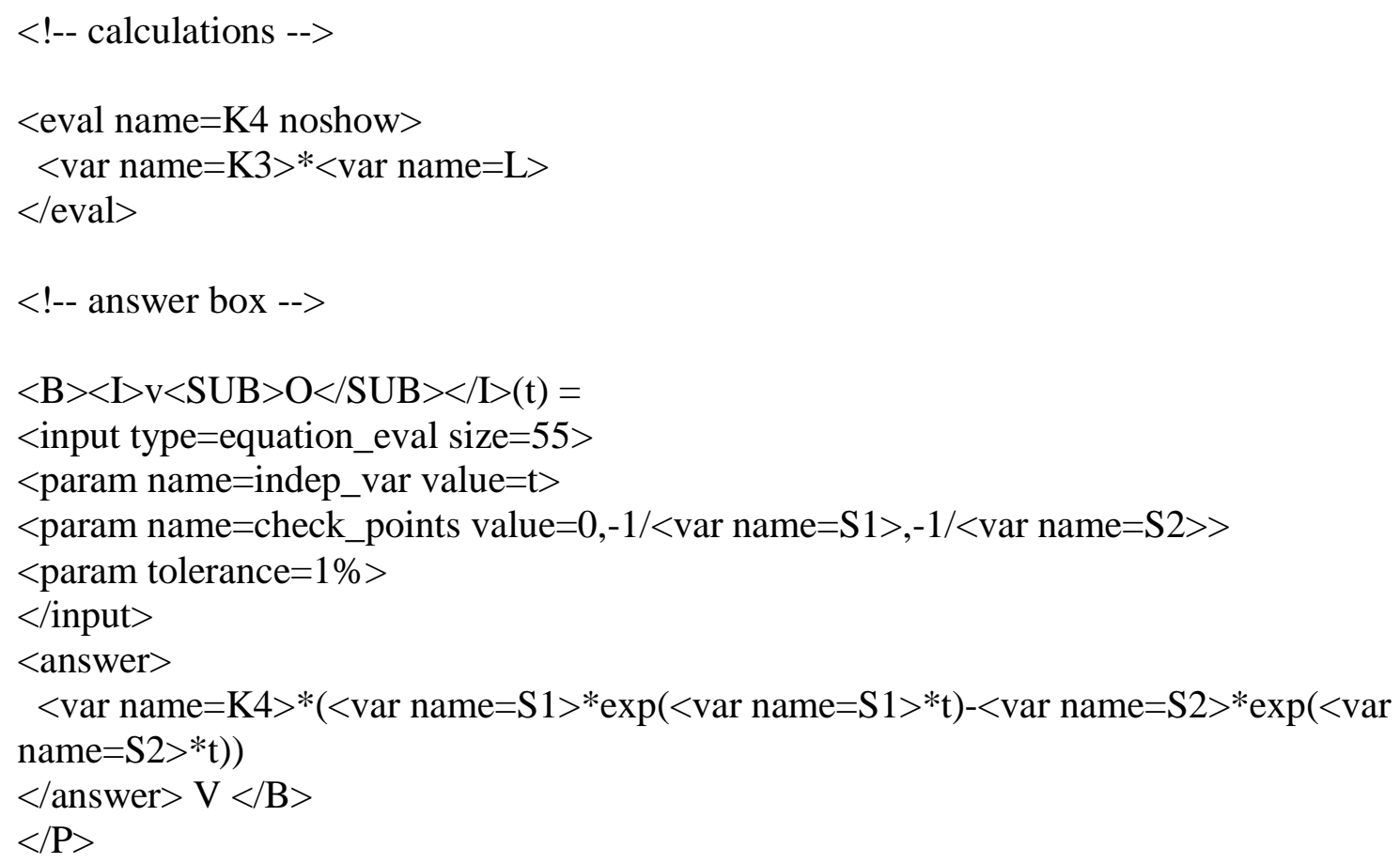

Browse Included and Linked Documents: test

Browse Included Images: Gteq.gif $>0$.

Bibliography

1. Mallard $\AA$ is a registered trademark of the University of Illinois. Mallard $®$ was developed by Professor Donna Brown and her associates in the Department of Electrical and Computer Engineering at Urbana IL. For additional information, see: http://www.cen.uiuc.edu/Mallard/

2. CircuitTutor ${ }^{\circledR}$ is a registered trademark of TutorWare, Inc., Champaign, IL.

3. Burks Oakley, II, "A Virtual Classroom Approach to Learning Circuit Analysis," IEEE Transactions on Education, vol.39, pp. 287-296, 1996.

4. Robert (Bob) M. Anderson, Jr., A First ALN Experience: Issues, Lessons and Emotions, Session 2432, Proceedings of the Annual Conference and Exposition, American Society for Engineering Education, June 28-July 1, 1998.

5. Robert (Bob) M. Anderson, Jr. and Eric A Cheek, Sr., Electric Circuits via the Internet: Sharing and Extending, Session 3232, Proceedings of the Annual Conference and Exposition, American Society for Engineering Education, June 20-23, 1999.

6. Roland E. Thomas and Albert J. Rosa, The Analysis and Design of Linear Circuits, Second Edition, Prentice Hall, 1998.

\section{HANS H. KUEHL}

Hans H. Kuehl received his B.S. from Princeton University and his and Ph.D. from Caltech, in Electrical Engineering. He has received the USC Associates Award for Teaching Excellence, the highest teaching award at the University of Southern California. His research interests include wave-plasma interactions. He is Professor and Associate Chairman of the Electrical Engineering-Electrophysics Department at USC. 